\title{
Electrical Versus Optical: Comparing Methods for Detecting Terahertz Radiation Using Neon Lamps
}

\author{
L. L. Slocombe ${ }^{1} \cdot$ R. A. Lewis ${ }^{2}$ (D)
}

Received: 24 November 2017 / Accepted: 2 May 2018 /

Published online: 18 May 2018

(C) The Author(s) 2018

\begin{abstract}
Terahertz radiation impinging on a lit neon tube causes additional ionization of the encapsulated gas. As a result, the electrical current flowing between the electrodes increases and the glow discharge in the tube brightens. These dual phenomena suggest two distinct modes of terahertz sensing. The electrical mode simply involves measuring the electrical current. The optical mode involves monitoring the brightness of the weakly ionized plasma glow discharge. Here, we directly compare the two detection modes under identical experimental conditions. We measure 0.1$\mathrm{THz}$ radiation modulated at frequencies in the range $0.1-10 \mathrm{kHz}$, for lamp currents in the range $1-10 \mathrm{~mA}$. We find that electrical detection provides a superior signal-tonoise ratio while optical detection has a faster response. Either method serves as the basis of a compact, robust, and inexpensive room-temperature detector of terahertz radiation.
\end{abstract}

Keywords Terahertz $\cdot \mathrm{THz} \cdot$ Neon tube $\cdot$ Neon lamp · Glow discharge detector · GDD · Plasma · Weakly ionized plasma - WIP · Detection · Detector · Sensing · Sensor · Upconversion · Responsivity · Signal-to-noise-ratio $\cdot$ SNR ·

Noise equivalent power $\cdot$ NEP

L. L. Slocombe

1s00338@surrey.ac.uk

R. A. Lewis

roger@uow.edu.au

1 Department of Physics, University of Surrey, Guildford GU2 7XH, UK

2 Institute for Superconducting and Electronic Materials and School of Physics, University of Wollongong, Wollongong, New South Wales 2522, Australia 


\section{Introduction}

Terahertz (THz) detectors that operate at room temperature tend to be slow, fragile, or expensive. Existing commercial room-temperature $\mathrm{THz}$ detectors include Golay cells, pyroelectric sensors, and Schottky diodes [1]. The Golay cell is fragile. The Golay cell and pyroelectric sensors have relatively long response times $\left(t_{\mathrm{r}} \approx 1 \mathrm{~s}\right.$ and $t_{\mathrm{r}} \approx 10 \mathrm{~ms}$, respectively). All three detectors have excellent sensitivity, but are expensive $\left(\approx 10^{2}-10^{4}\right.$ USD), a serious shortcoming for widespread application in industry. In contrast, a miniature neon lamp (also called a neon tube) may be exploited as a relatively inexpensive $(\approx 1 \mathrm{USD})$ as well as relatively fast $\left(t_{\mathrm{r}} \approx 1 \mu \mathrm{s}\right)$ glow discharge detector (GDD) of $\mathrm{THz}$ radiation. Moreover, the neon lamp is compact and robust. Key parameters of these four room-temperature $\mathrm{THz}$ detectors are summarized in Table 1. In this work, we compare two different modes of monitoring the plasma discharge in a neon lamp to sense $\mathrm{THz}$ radiation.

The use of plasma discharges to detect microwave radiation is well established, having been reported since the 1950s [6]. The plasmas in commercially available neon lamps have been found suitable for this purpose. Neon lamps have been used for detection not only in the microwave region, but also in the THz, IR, and UV bands $\left(10^{9}-10^{15} \mathrm{~Hz}\right)[7,8]$. This is a remarkably broad range, spanning six orders of magnitude. Furthermore, neon lamps as GDDs exhibit a wide dynamic range [9], are electronically rugged (can be exposed up to $30 \mathrm{~kW}$ of light without damage) [10], and exhibit a relatively low noise equivalent power (NEP) $\left(10^{-8}\right.$ to $\left.10^{-12} \mathrm{WHz}^{-1 / 2}\right)$ $[4,5]$.

The well-established operation of the neon lamp will be presented as a prelude to describing the operation of the lamp as a GDD. Neon lamps demonstrate distinct domains in their current voltage characteristic, as illustrated in Fig. 1 for the RS 655-9413 lamp. Each region is rich in physics. Each region emits a different optical spectrum (Fig. 1a) and exhibits a different visual appearance (Fig. 1b) corresponding to a different current-voltage dependence (Fig. 1c). For a more complete introduction to the various operating IV regions, see ref. [4]. The "abnormal" region is where the largest current flows before breakdown begins in the "arc" region. Thus, most reported applications of neon lamps as GDDs utilize the "abnormal" region. In practice, this corresponds to an applied current on the order of $\approx 1 \mathrm{~mA}$, corresponding to a lamp voltage of $\approx 60 \mathrm{~V}$. This is well above the rated working current of $0.25 \mathrm{~mA}$.

Table 1 Room-temperature terahertz (THz) detectors. The neon lamp is a type of glow discharge detector (GDD). The responsivity provides a metric for the given output per signal input. All data are expressed as order of magnitude

\begin{tabular}{lllll}
\hline Type & Cost $(\$)$ & $\begin{array}{l}\text { Responsivity } \\
\mathrm{R}\left(\mathrm{VW}^{-1}\right)\end{array}$ & Size $\left(\mathrm{cm}^{3}\right)$ & Reference \\
\hline Pyroelectric & $10^{2}$ & $10^{3}$ & 1 & {$[2]$} \\
Golay & $10^{4}$ & $10^{5}$ & $10^{2}$ & {$[3]$} \\
Schottky & $10^{4}$ & $10^{2}$ & $<1$ & {$[4]$} \\
GDD & 1 & $10^{1}$ & $<1$ & {$[4,5]$} \\
\hline
\end{tabular}




\section{a) Optical emission}
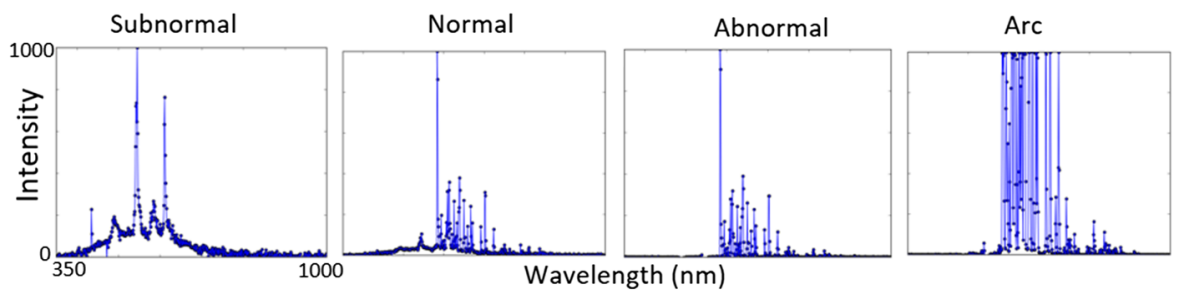

\section{b) Image}
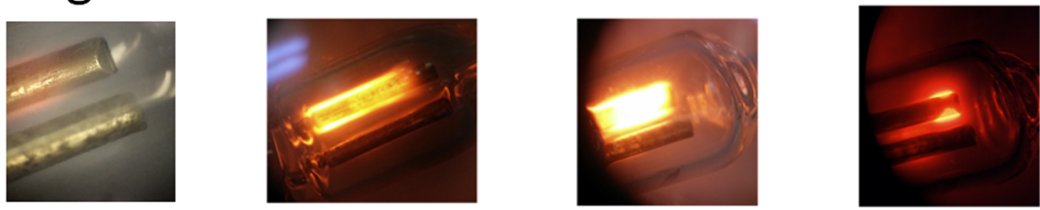

\section{c) I-V curve}

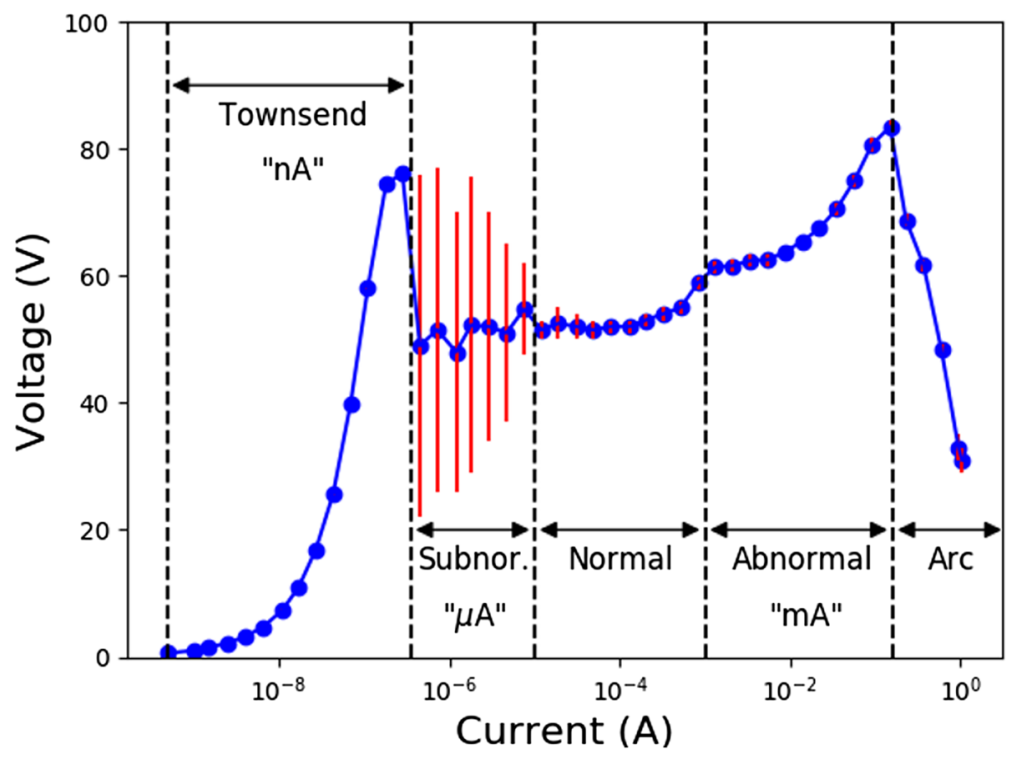

Fig. 1 Overview of the operating states of a neon lamp (RS 655-9413): Townsend, subnormal, normal, abnormal, and arc. The regions are illustrated using $\mathbf{a}$ UV-Vis spectroscopy, b visual appearance, and $\mathbf{c}$ I-V characteristic. The Townsend region displays no optical phenomena; the subnormal image is captured mid flash. Error bars indicate the range of fluctuation when the lamp is acting as a relaxation oscillator

If THz radiation falls on a neon lamp operating in the "abnormal region," several things occur. The plasma in the lamp is already weakly ionized by the DC source. This plasma absorbs $\mathrm{THz}$ radiation, thus becoming further ionized. This increases the current flowing in the lamp. In addition, this increases the brightness of the glow discharge. In this sense, the terahertz radiation is "up-converted" to produce an 
electromagnetic response at a higher frequency, in the visible. The change in visible radiation intensity may be measured with a suitable visible detector, such as a Si photodiode (PD). In the next section, this qualitative overview is put on a firmer theoretical foundation.

\section{Theory}

The dominant detection mechanism of radiation by a GDD is enhanced cascade ionization [11-13]. The incident electromagnetic radiation slightly increases the energy of the electrons in the negative glow region via inverse-bremsstrahlung heating [14]. The increase in energy is sufficient to convert a fraction of excitation collisions into ionization collisions, thus increasing the current density in the plasma. The DC electric field between the electrodes accelerates the electrons to generate an avalanche effect, with a gain estimated to be on the order of $10^{6}$ [15]. Competing with this is enhanced electron diffusion, as electrons in the Faraday dark space are heated by the incoming THz, increasing the average migration of electrons to the glass walls. Incident beam diameters which are larger than the interelectrode gap result in a measured signal which is a combination of the two effects [13].

It has been shown that the change in bias current as a function of time due to an incident radiation field triggering an enhanced cascade is given by [15]:

$$
\Delta I(t)=\frac{G v_{e} n_{e} e^{2}}{V_{\text {ion }} m_{e}}\left(\frac{\tau}{\tau_{\text {ion }}}\right) \eta_{0} P_{\mathrm{D}}\left(\frac{v}{v^{2}+\omega^{2}}\right)\left(1-e^{-t / \tau}\right) .
$$

Here, $e$ is the charge on the electron and $v_{e}, n_{e}$, and $m_{e}$ are the electron velocity, number density, and mass, respectively. $V_{\text {ion }}$ is the gas ionization potential, $\eta_{0}$ is the impedance of free space, $\omega$ is the incident radiation frequency, and $P_{\mathrm{D}}$ is the radiation power density; $\tau$ is the induced current response time, $\tau_{\text {ion }}$ is the ionizing time, and $v$ is the ionization collision frequency. The internal signal gain, $G$, is expressed as:

$$
G \approx \frac{\exp \left(2 v t_{\mathrm{d}}\right)}{2 v t_{\mathrm{d}}}
$$

where $t_{\mathrm{d}}$ is the average electron drift time to the anode. In the case when the other parameters are not varying with time, and at sufficiently delayed measurement times $(t \gg \tau)$, the change in current is directly proportional to the incident radiation power.

On the other hand, the change in current induced by enhanced diffusion is given by:

$$
\Delta I(t)=\Delta v \times n_{e}+v \Delta n_{e}-\Delta D \times \nabla^{2} n_{e}-D \times \nabla^{2}\left(\Delta n_{e}\right),
$$

where $D$ is the diffusion coefficient.

One significant disadvantage of electrical detection is that the response is limited by the electric circuit used to determine $\Delta I(t)$. This circuit may be divided into two parts: the equivalent circuit parameters of the lamp and the external circuitry. Calculations indicate that the response time of the plasma itself is on the order of 
picoseconds [15]. However, the lamp AC equivalent circuit involves parasitic capacitance and inductance that significantly increase the response time to the order of microseconds [15]. The capacitance arises from the charge at the electrodes and the potential difference between them. The inductance derives from the magnetic flux generated by the movement of the charged species in the plasma. In addition to these phenomena in the lamp, the external circuit will slow the detection further. On the other hand, optical detection, for example, by a Si PD, can be much faster, as the response time is limited by the PD circuit instead. A second significant disadvantage of electrical detection is the electrical shot noise arising from the fluctuations in current from the cathode. Observing the optical emission instead of the electrical current also decouples this noise from the measurement.

Optical techniques have been used to monitor the visible fluctuations induced by $\mathrm{THz}$ in a large positive column slab, using a CCD camera, with a response time of $0.8 \pm 0.1 \mu \mathrm{s}[16,17]$. Other applications employ a Si PD [18, 19]. The specific model is a Thorlabs PDB210A/M balanced amplified PD consisting of two matched PDs with a low-noise, high-speed transimpedance amplifier (TIA) [20]. The cost of this unit is $\approx 10^{3}$ USD, considerably more than the base cost of the neon lamp. In contrast, the work we report here utilizes a relatively inexpensive optical detector $(\approx 10 \mathrm{USD})$, so located in the space of providing a relatively inexpensive roomtemperature detector of $\mathrm{THz}$ radiation.

\section{Experiment}

\subsection{Equipment}

The experimental arrangement is presented in Fig. 2. The $\mathrm{THz}$ source was an IMPATT diode (Terasense Type-X) emitting at $0.1 \mathrm{THz}$ with power of $\approx 80 \mathrm{~mW}$. The $\mathrm{CW} \mathrm{THz} \mathrm{beam} \mathrm{output} \mathrm{is} \mathrm{modulated} \mathrm{electrically} \mathrm{using} \mathrm{a} \mathrm{function} \mathrm{generator} \mathrm{(TG120}$ $20 \mathrm{MHz}$ Thurlby Thandar Instruments) producing a square wave over a range of frequencies from $100 \mathrm{~Hz}$ to $10 \mathrm{kHz}$.

Initial screening of a number of neon lamps (104-777, 105-017, 655-9407, 6559413, 655-96-9) from the supplier RS indicated that the RS 655-9413 was the best of the selection. This was then used for all the work reported here. The neon lamp was biased by a power supply (Keithley 2400) acting as a current source.

A lock-in amplifier (LIA; Stanford Research Labs SR830) was used to demodulate the detected signal. The output of the LIA was fed to a voltmeter (Keithley 2000) to facilitate our RS-232 interfacing. Both the current source and voltmeter are controlled by a Python program via RS-232 protocol.

The optical method requires all the same experimental components and, in addition, a means of measuring the visible output of the neon lamp. For this, an OPT101 Monolithic PD and single-supply TIA was used [21]. The OPT101 was mounted to a custom-made circuit board. The common pin (8), $-\mathrm{V}$ (3) and the negative terminal of the cell were grounded. The module was powered by a dry $9 \mathrm{~V}$ cell. Both module and cell were mounted in a shielded box. The output signal was collected via a short coaxial cable. The shielding and short lead were employed to reduce noise. The 


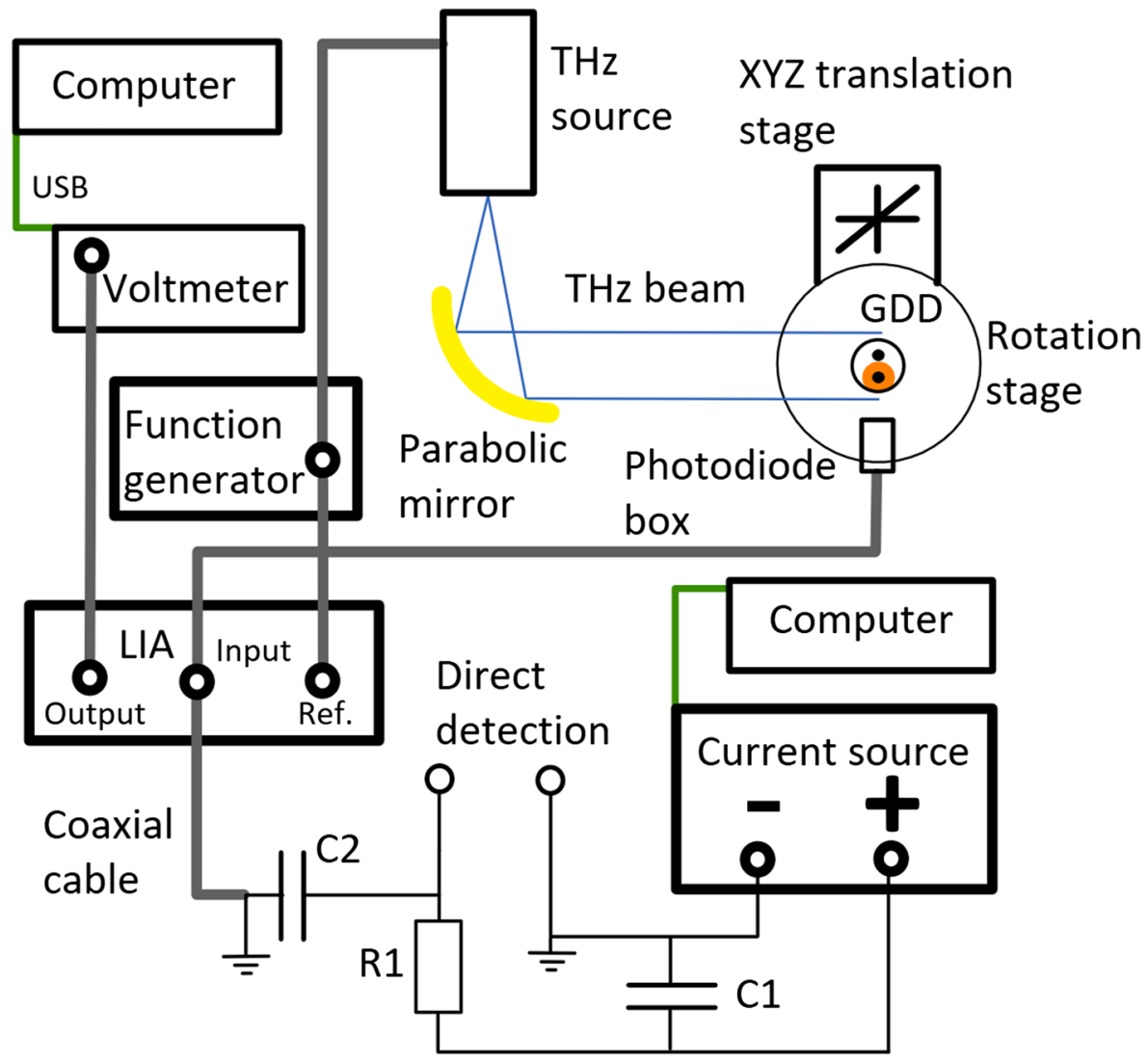

Fig. 2 Experimental configuration used to determine the current and the frequency dependence. The lowpass filter (R1 and $\mathrm{C} 1$ ) attenuates the noise of the power supply. $\mathrm{C} 1$ capacitively couples the GDD to the LIA. The THz beam is larger than the GDD; we estimate that $5 \mathrm{~mW}$ intersects the GDD cross section. The voltmeter was used in addition to the LIA for ease of programmability

internal feedback resistor and capacitor were used [21]. The feedback resistor governs the output voltage. In the ideal case, $V_{\text {out }}=I_{\mathrm{p}} R_{\mathrm{F}}$. The feedback capacitor reduces the effects of gain peaking and ringing.

The PD box was orientated to face the negative glow (at the negative terminal), where most of the light is emitted. In all measurements reported here, the face of photodiode was positioned at $2 \mathrm{~cm}$ from the center of the neon lamp. The PD picks up the integrated spectral output of the GDD between 400 and $1100 \mathrm{~nm}$ [21].

\subsection{Methods}

There are several metrics by which to compare the electrical and optical detection schemes. Two are defined here, responsivity and signal-to-noise ratio.

The responsivity, $R$, is a measure of the efficiency of a detector, that is, the desired output for the given input. Here, the input is the THz power falling onto the lamp, $P$ 
(measured in watts). The output, $A$, is the voltage produced by the detector (measured in volts).

$$
R=\frac{A}{P} \text {. }
$$

(The responsivity may also be thought of as the "gain" of the detector.) Generally, high responsivity is preferred. While the definition of $R$ is straightforward, care needs be exercised in how both denominator and numerator are defined as different workers use different conventions. With respect to the input, the power might be the power emitted by the source, or that portion that is focussed onto the neon lamp, or that portion which is transmitted through the glass envelope of the lamp into the plasma itself. (The THz transmission of the glass envelope has been reported by Hou et al. [4].) Here, we take a conservative approach by employing the total power falling on the GDD cross section, estimated to be $5 \mathrm{~mW}$. With respect to the output, the value of $A$ will be influenced by any amplification steps. We again take a conservative approach and correct for amplification in the electrical method, and for the TIA and other amplification stages in the optical method, to give the voltage produced directly across either the neon lamp or the PD, respectively. In view of this discussion, caution needs to be taken in comparing absolute values of $R$ between reports. However, relative changes, for example, as a function of lamp voltage, should be comparable.

As well as the desired signal, the output of the detector contains spurious signal, or noise. We define the noise to be the standard deviation of the signal, $\sigma$. The mean of the output signal is denoted $\bar{A}$. Then, the signal-to-noise-ratio (SNR) is:

$$
\mathrm{SNR}=\frac{\bar{A}}{\sigma} .
$$

A high value of SNR is desirable.

\section{Current Dependence}

This section investigates the response and the SNR as a function of the current supplied to the GDD, comparing the electrical and optical detection methods. The experimental data are presented in Fig. 3.

\subsection{Responsivity}

\subsubsection{Electrical Detection}

At currents in the nanoamp and microamp ranges, corresponding to the Townsend and subnormal regions, few atoms are ionized. Thus, there is very little current flow, leading to a negligible response to $\mathrm{THz}$ radiation [4].

As the current is increased to the milliamp range, corresponding to the the normal and abnormal glow regions, there is a significant population of excited neon atoms. Thus, the incident $\mathrm{THz}$ photons can increase the ionization rate of the gas. The newly generated electrons increase the current across the lamp producing an approximately linear response, as described by Eq. 1, and observed in Fig. 3. 

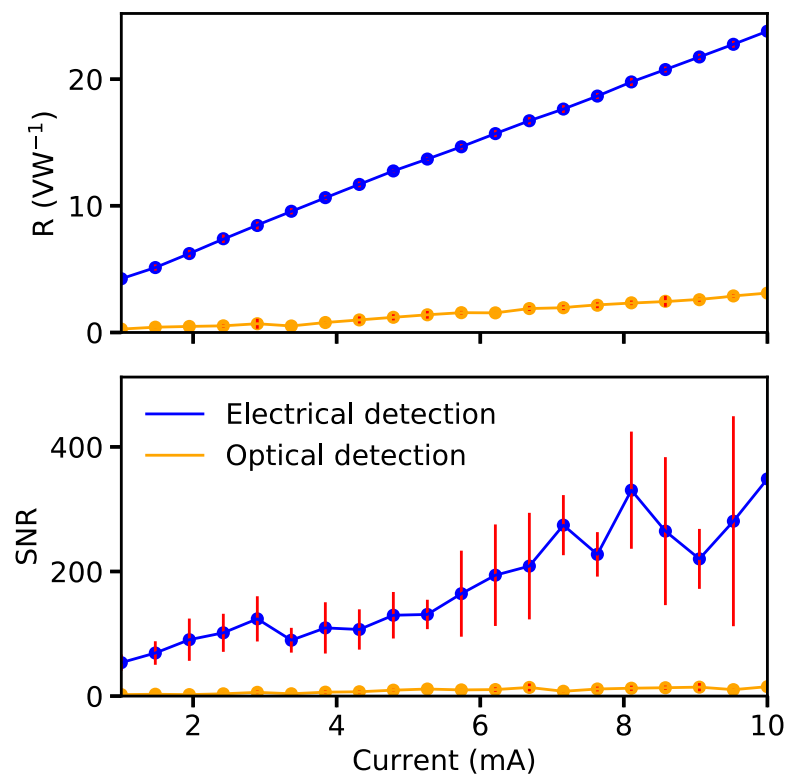

Fig. 3 Responsivity and signal-to-noise ratio as a function of current for RS 655-9413 neon lamp under $5 \mathrm{~mW}$ of $0.1 \mathrm{THz}$ radiation at modulation frequency of $1 \mathrm{kHz}$. The current source was swept upwards. For each measurement, 100 data points are recorded, allowing the mean and standard deviation to be calculated. The time constant of the LIA was set to $100 \mathrm{~ms}$ with a $12 \mathrm{~dB} /$ oct roll-off. Each data point shown is an average of 5 repeats of different lamps. Error bars indicate one $\sigma$ of variation across 5 different lamps

The dependence of the responsivity with current that we observe for the neon lamp RS 655-9413 is very similar to that observed for neon lamp NE-2B-3x8 [4] and for neon lamp N523 [18, 19].

While increasing the current offers a higher response, operating past the midsection of the abnormal glow region results in permanent damage to the lamp, caused by sputtering. Increasing the current still further, the GDD becomes increasingly unstable, and the reliable detection lifetime reduces to a matter of minutes [22]. For reasonable stability and lifetime, the lamp should be operated below $10 \mathrm{~mA}$.

\subsubsection{Optical Detection}

We observe a linear increase in responsivity with current for optical detection (Fig. 3). An increase in responsivity with current has previously been reported for the neon lamp N523 [18, 19]. In that case, a superlinear response was reported. This response is understandable given that the N523 lamp has a phosphor coating, in contrast to the RS 655-9413 lamp that has no such coating. The phosphor coating introduces an additional pathway for further photoexcitation by the fluorescent photons. The additional pathway feeds back into the gain mechanism, further enhancing the signal [23] and so leading to a non-linear response. 


\subsubsection{Comparison}

We observe that the electrical responsivity is better than the optical responsivity at all currents and the ratio between the responsivities does not vary much with current, always being $\approx 5 \times$. This result is in contrast with earlier work on a different lamp where the optical signal was found to be about $\approx 2 \times$ as strong as the electrical signal at a current of $2 \mathrm{~mA}$, increasing to $\approx 3 \times$ as strong at a current of $10 \mathrm{~mA}[18,19]$. However, in the earlier work, while it is specified the electrical detection mode was without amplification, it is not clear what gain setting was used on the TIA in the optical detection mode. The TIA gain can be adjusted by $R_{\mathrm{F}}$ to an upper limit (until bandwidth and instability issues begin to dominate). Increasing $R$ also results in a decrease in thermal noise, as this scales as $1 / \sqrt{R_{\mathrm{F}}}$. Another factor in considering the responsivity of the optical method is that the light output from the lamp is almost isotropic, so much of the signal is not collected by the PD. Consequently, the signal could be increased by introducing appropriate mirrors and lenses to collect a greater fraction of the light however made little improvement to the SNR. Regardless of the differences in detail between the two experiments, the relative sizes of the electrical and optical responsivities are never vastly different, always within an order of magnitude. The current dependence of the RS 655-9413 lamp is fairly repeatable for both methods, as the error bars demonstrate in indicating the fluctuation across 5 lamps. Non-repeatability is attributed to non-uniformity in the manufacturing quality control.

\subsection{Signal-to-Noise Ratio}

We observe that the SNR for electrical detection generally increases as current increases over the range 1-10 mA. This is consistent with work reported on the NE2B-3x8 neon lamp, where the noise power spectral density $\left(S_{v}\right)$ was found to decrease with increasing current over the steps 3.0, 5.3, 6.7, 9.0, and 10.2 $\mathrm{mA}$ [4].

Likewise, we observe that the SNR for optical detection generally increases as current increases over the range $1-10 \mathrm{~mA}$, although the data is more fluctuating than in the electrical detection case.

Thus, our observation is that the SNR for electrical detection is much better than for optical detection over the whole current range employed. We attribute this to the higher responsivity of the electrical method, rather than greater noise in the optical method.

In contrast, a report on the N523 neon lamp measures a noise voltage of $130 \mathrm{nV}$ $\mathrm{Hz}^{-1 / 2}$, corresponding to a noise equivalent power of about $1.3 \mathrm{nW} \mathrm{Hz}{ }^{-1 / 2}$, superior to the electrical detection figure of about $10 \mathrm{nW} \mathrm{Hz}^{-1 / 2}$ measured on the same lamp previously $[18,19]$.

\section{Frequency Dependence}

This section investigates the quality of the signal with respect to the modulation frequency of the $\mathrm{THz}$ radiation. In general, in view of $1 / f$ noise, it would be expected 
that operating at higher modulation frequency would be preferred, until the response time becomes significant. The experimental data are presented in Fig. 4.

\subsection{Responsivity}

The electrical responsivity is fairly uniform over the frequency range of 100$10,000 \mathrm{~Hz}$, then exhibits a resonance peak at about $40 \mathrm{kHz}$, rolling off to the maximum frequency measured, $100 \mathrm{kHz}$. The optical responsivity is also fairly flat over the range $100-10,000 \mathrm{~Hz}$ and also exhibits a resonance at around $40 \mathrm{kHz}$. The optical method demonstrates a roll-off just before the resonance. We observe that the electrical responsivity exceeds the optical responsivity at all frequencies. As the frequency increases, the electrical responsivity becomes relatively better. This is attributable to the PD detector module having a bandwidth of only $14 \mathrm{kHz}$. This not only starts to affect the responsivity at lower frequencies, but also suppresses the resonance at $40 \mathrm{kHz}$.

The origin of the resonance observed at $40 \mathrm{kHz}$ is not presently known with certainty but is the subject of further investigation. The resonance is not caused by the external electrical circuitry, as the electrical and optical methods use different values of inductance, capacitance, and resistance. Thus, the resonance must originate in the plasma. Previous work on the N523 and N527 neon lamps over the range $1-90 \mathrm{kHz}$ did not observe any resonance in the detected signal, but rather an increase in signal with frequency [24]. On the other hand, other works (on an unidentified tube) over
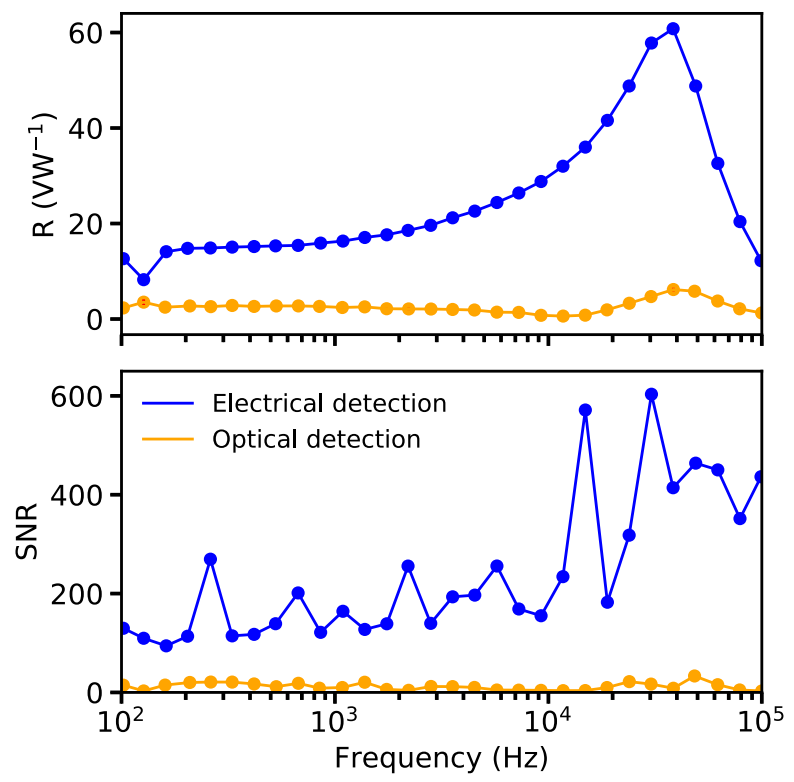

Fig. 4 Responsivity and signal-to-noise ratio as a function of radiation modulation frequency for RS 6559413 neon lamp under $5 \mathrm{~mW}$ of $0.1-\mathrm{THz}$ radiation. The current in the lamp was $10 \mathrm{~mA}$. The same LIA configuration was employed as in section "4." The lower modulation frequency was set at $100 \mathrm{~Hz}$ to avoid pickup from the electrical mains. The upper modulation frequency was limited by the range of the LIA 
the modulation frequency range $100-6000 \mathrm{~Hz}$ show a clear resonance between $500-$ $600 \mathrm{~Hz}$, but did not give a full account of the origin of the resonance [25].

\subsection{Signal-to-Noise Ratio}

While the SNR shows some fluctuation for electrical detection, it is relatively uniform in the range $100-10,000 \mathrm{~Hz}$. It then increases in the range $10-100 \mathrm{kHz}$, which reflects the increase in responsivity in this range. These observations are consistent with work reported on the NE-2B-3x8 neon lamp, where the noise power spectral density $\left(S_{v}\right)$ was found to exhibit little variation in the range $1-100 \mathrm{kHz}$ when the lamp was operated at $10.2 \mathrm{~mA}$ [4]. For optical detection, the SNR is uniformly low over the whole frequency range measured. Thus, under the experimental conditions we have employed, electrical detection gives much better SNR over the entire frequency range.

\section{Rise Time}

We have measured the rise time of the signal using an SDS1000DL Oscilloscope. We find that the rise time for electrical detection is $22 \pm 1 \mu \mathrm{s}$ and the rise time for optical detection is $12.6 \pm 0.7 \mu \mathrm{s}$. Thus, the optical detection method is about twice as fast as the electrical detection method. This result is consistent with an earlier report for neon lamp N523, which also found the optical method to be about twice as fast as the electrical method $[18,19]$. However, the absolute values differ.

\section{Summary}

We have compared in detail two methods of detecting $\mathrm{THz}$ radiation using neon lamps. Here, we draw together our findings in summary form.

Simplicity The electrical detection method is direct and simple. The optical method is more complicated, involving in addition to the neon lamp an optical sensor. Both methods share the need to modulate the radiation (typically with mechanical chopping or electrical modulation), to amplify the detector output (typically with a band-filtering amplifier) and to demodulate the signal (typically with a lock-in amplifier).

Size The neon lamp is relatively compact, of the order of $1 \mathrm{~cm}^{3}$. Adding the simplest PD approximately doubles the size. Adding a more sophisticated PD, such as found in a balanced system, increases the size by approximately an order of magnitude.

Cost The neon lamp at the heart of both detection schemes is of the order of 1 USD. The simplest optical systems, such as the OPT101 module, add a cost of the order of 10 USD. More sophisticated photodetectors, such as the PDB210A/M balanced amplified PD, add a cost of the order of 1000 USD. These are the costs pertinent in differentiating the detection systems; the entire cost of the detection system also 
includes the cost of modulation and demodulation (as would also be the case with, for example, a Schottky diode).

Responsivity Care needs to be taken in defining the terms in the responsivity equation. The voltage delivered by the detector depends on the operating current and frequency as well as any included electronic amplification. The power delivered may be defined as the total power of the source, or some fraction of that. Over a range of currents and frequencies, and regardless of how the power is defined, we have found that electrical detection provides better responsivity than optical detection. (However, others, using a different lamp, have found the opposite.)

Noise The noise in the entire detection system has many sources. We have found, as have previous workers, that the absolute noise decreases with current and decreases with frequency. We have also found that optical detection, even with an inexpensive PD, can successfully decouple noise, parasitic capacitance, and inductance. Thus, the noise present in optical detection is lower than that in electrical detection. However, since the responsivity for the optical system is much lower than that for the electrical system, electrical detection gives a better SNR than optical detection.

Rise Time The fundamental changes in the plasma as a result of $\mathrm{THz}$ irradiation take place on a picosecond time scale. For practical use, these changes have to be read by external circuits. As others have observed, we find that the optical method results in a faster response (or smaller rise time) than the electrical method.

\section{Conclusion}

Both electrical detection and optical detection serve as practical detectors of $\mathrm{THz}$ radiation. The electrical mode offers a detector that is smaller, simpler, and less expensive than the optical mode and (in the case of the neon lamp measured here) with higher responsivity and higher SNR. On the other hand, the optical mode offers a faster detector, having smaller rise time and thus greater bandwidth.

Acknowledgements The authors thank Peter Ihnat for his valuable and tireless assistance in relation to the detection circuits, Colin Bleasdale for preliminary measurements, Josip Horvat and Enbang Li for useful discussions and Stephen Sweeney for reading the manuscript.

Funding Information This work was supported by the Australian Research Council and by the University of Wollongong Faculty of Engineering and Information Sciences.

Open Access This article is distributed under the terms of the Creative Commons Attribution 4.0 International License (http://creativecommons.org/licenses/by/4.0/), which permits unrestricted use, distribution, and reproduction in any medium, provided you give appropriate credit to the original author(s) and the source, provide a link to the Creative Commons license, and indicate if changes were made.

\section{References}

1. R.A. Lewis, in Encyclopedia of Spectroscopy and Spectrometry, 3rd Edition, vol 4, ed. by J. Lindon, G. Tranter, D. Koppenaal (Elsevier, Oxford, 2017), pp. 422-426. 
2. Y. Lee, Principles of Terahertz Science and Technology. Lecture Notes in Physics (Springer, 2009).

3. M.J.E. Golay, Review of Scientific Instruments 18, 347 (1947).

4. L. Hou, H. Park, X.C. Zhang, IEEE Journal of Selected Topics in Quantum Electronics 17(1), 177 (2011).

5. N. Kopeika, International Journal of Infrared and Millimeter Waves 5(9), 1333 (1984).

6. G. Burroughs, A. Bronwell, Tel-Tech. 11, 62 (1952).

7. P. Severin, A. Van Nie, IEEE Transactions on Microwave Theory and Techniques 14(9), 431 (1966).

8. L. Hou, H. Park, X.C. Zhang, in Infrared, Millimeter, and Terahertz Waves, 2009. IRMMW-THz 2009. 34th International Conference on (IEEE, 2009), pp. 1-2.

9. N.S. Kopeika, N.H. Farhat, IEEE Transactions on Electron Devices 22(8), 534 (1975).

10. A. Golan, M. Einat, IEEE Transactions on Electron Devices 64(6), 2670 (2017).

11. L. Hou, W. Shi, Y. Wu, H. Liu, Y. Ding, in Infrared, Millimeter, and Terahertz Waves (IRMMW-THz), 2013 38th International Conference on (IEEE, 2013), pp. 1-2.

12. N. Kopeika, Proceedings of the IEEE 63(6), 981 (1975).

13. D. Rozban, N.S. Kopeika, A. Abamovich, E. Farber, Journal of Applied Physics 103(9), 093306 (2008).

14. J. Liu, X.C. Zhang, Frontiers of Optoelectronics 7(2), 156 (2014).

15. N. Kopeika, IEEE Transactions on Plasma Science 6(2), 139 (1978).

16. M.S. Gitlin, Plasma Physics Reports 43(2), 253 (2017).

17. M.S. Gitlin, M.Y. Glyavin, A.E. Fedotov, A.I. Tsvetkov, Plasma Physics Reports 43(7), 778 (2017).

18. A. Aharon (Akram), D. Rozban, A. Abramovich, Y. Yitzhaky, N.S. Kopeika, in SPIE Security+ Defence (International Society for Optics and Photonics, 2016), pp. 999,309-999,309.

19. A. Aharon (Akram), D. Rozban, A. Klein, A. Abramovich, Y. Yitzhaky, N.S. Kopeika, Photon. Res. 4(6), 306 (2016).

20. Thorlabs, Large-Area Balanced Photodetector (2014). PDB210A/M, Rev. 2.1.

21. Texas Instruments, Monolithic Photodiode and Single-Supply Transimpedance Amplifier (2015). OPT-101, Rev. B.

22. D. Rozban, A.A. Akram, A. Abramovich, N.S. Kopeika, IEEE Sensors Journal 15(11), 6659 (2015).

23. A. Abramovich, N.S. Kopeika, D. Rozban, E. Farber, Appl. Opt. 46(29), 7207 (2007).

24. N. Alasgarzade, M.A. Nebioğlu, T. Takan, I.U. Uzun-Kaymak, A.B. Sahin, H. Altan, in THz for CBRN and Explosives Detection and Diagnosis, ed. by M.F. Pereira, O. Shulika (Springer Netherlands, Dordrecht, 2017), pp. 179-187.

25. K. Çınar, H. Altan, A.B. Şahin, in Proc. SPIE 8716 (2013), pp. 8716 - 8716 - 9. 